This item was submitted to Loughborough's Research Repository by the author.

Items in Figshare are protected by copyright, with all rights reserved, unless otherwise indicated.

\title{
Facile production of biodegradable bipolymer patchy and patchy Janus particles with controlled morphology by microfluidic routes
}

\section{PLEASE CITE THE PUBLISHED VERSION}

http://dx.doi.org/10.1021/acs.langmuir.7b02506

\section{PUBLISHER}

American Chemical Society

\section{VERSION}

VoR (Version of Record)

\section{PUBLISHER STATEMENT}

This work is made available according to the conditions of the Creative Commons Attribution 4.0 International (CC BY 4.0) licence. Full details of this licence are available at: http://creativecommons.org/licenses/ by/4.0/

\section{LICENCE}

CC BY 4.0

\section{REPOSITORY RECORD}

Ekanem, Ekanem E., Zilin Zhang, and Goran Vladisavljevic. 2017. "Facile Production of Biodegradable Bipolymer Patchy and Patchy Janus Particles with Controlled Morphology by Microfluidic Routes". figshare. https://hdl.handle.net/2134/26106. 


\title{
Facile Production of Biodegradable Bipolymer Patchy and Patchy Janus Particles with Controlled Morphology by Microfluidic Routes
}

\author{
Ekanem E. Ekanem, Zilin Zhang, and Goran T. Vladisavljević**0 \\ Department of Chemical Engineering, Loughborough University, Loughborough LE11 3TU, U.K.
}

Supporting Information

ABSTRACT: Patchy and patchy Janus particles composed of poly(DL-lactic acid) (PLA) and polycaprolactone (PCL) regions were produced with a controlled size, patchiness, composition, and shape anisotropy by microfluidic emulsification and solvent evaporation. Isotropic particles composed of PCL patches embedded in the PLA matrix were produced from relatively small drops with a diameter of 14-25 $\mu \mathrm{m}$ because of the fast solvent extraction as a result of high interfacial area of the particles. Anisotropic patchy Janus particles were formed from large drops, $100-250 \mu \mathrm{m}$ in diameter. A higher degree of polymer separation was achieved using a higher ratio of dichloromethane to ethyl acetate in the organic phase because of the more pronounced patch coarsening via Ostwald ripening. Janus particles with two fully separated polymer compartments were produced by in situ microfluidic mixing of two separate polymer streams within the formed droplets. The advantage of in situ micromixing is that the particle morphology can be changed continuously in a facile manner during drop generation by manipulating the organic stream flow rates. PCL and PLA domains within the particles were visualized by confocal laser scanning microscopy because of the preferential adsorption of rhodamine 6G dye onto PLA domains and higher binding affinity of Nile red toward PCL.

\section{INTRODUCTION}

Microfluidics deals with the manipulation of fluids at the microscale level. Because of laminar flow, reducing axial dispersion effects and achieving good mixing in such systems are challenging. Early studies dealt with reducing the mean diffusive path by hydrodynamic flow focussing ${ }^{1}$ or chaotic advection. ${ }^{2}$ Later works involved segmented flows where a second phase was introduced to a continuous fluid stream. The segments were characterized by constant residence time that led to narrow particle size distributions when such segmented flows were used for particle synthesis. ${ }^{3}$ To prevent intrasegment contamination when precipitous or gelatinous products were formed, segmented flow systems evolved into drop microfluidic systems. ${ }^{4}$ In these systems, micromixing was achieved within the continuously generated drops, which served as microchambers for mixing. In this approach, the precipitation of products onto the channel walls was prevented because the drops do not wet the walls. Drops generated in microfluidic systems are increasingly used as templates for the production of functional particles.

Solvent evaporation/extraction from organic droplets creates a means for dissolved polymers to internally self-assemble into structured particles. ${ }^{6,7}$ The size, polydispersity, and structure of such particles depend on the properties of the parent drops. ${ }^{8,9}$ Depending on their intended use, microparticles are required to possess certain morphological characteristics.
Janus and patchy Janus particles have unique properties that are impossible to achieve with homogeneous materials, such as tuneable and controllable asymmetry in shape, composition, surface topology, and physical properties. 9 Patchy Janus particles can be made by adhering nanoparticles selectively onto one of the compartments of Janus particles through the covalent attachment, ${ }^{10}$ hydrogen bonding, ${ }^{11}$ or liquid-crystaldirected positioning, ${ }^{12}$ which often requires complex multistep processing. Recently, non-biodegradable patchy Janus particles composed of block copolymer/homopolymer blends were prepared using a simple solvent evaporation process. ${ }^{13}$

In this work, we have developed novel facile methods for the fabrication of patchy Janus particles made up of two synthetic biodegradable polymers [poly(DL-lactic acid) (PLA) and polycaprolactone (PCL)] using microfluidic emulsification and solvent evaporation. PLA and PCL are biocompatible polymers and, when used in therapeutic applications in vivo, do not exhibit any adverse local or systemic effects. ${ }^{14}$ PCL is highly hydrophobic and partially crystalline and thus has longer degradation times than the racemic PLA, which is amorphous. The difference in biodegradability of both polymers can be exploited for time-programmed dual-drug delivery. The objective of this study was to investigate the effects of emulsion

Received: July 18, 2017

Revised: July 31, 2017

Published: August 4, 2017 
formulation, droplet size, and fabrication route on the morphology of patchy/Janus particles.

Figure 1 shows the changes in the droplet morphology caused by solvent evaporation from a droplet composed of a

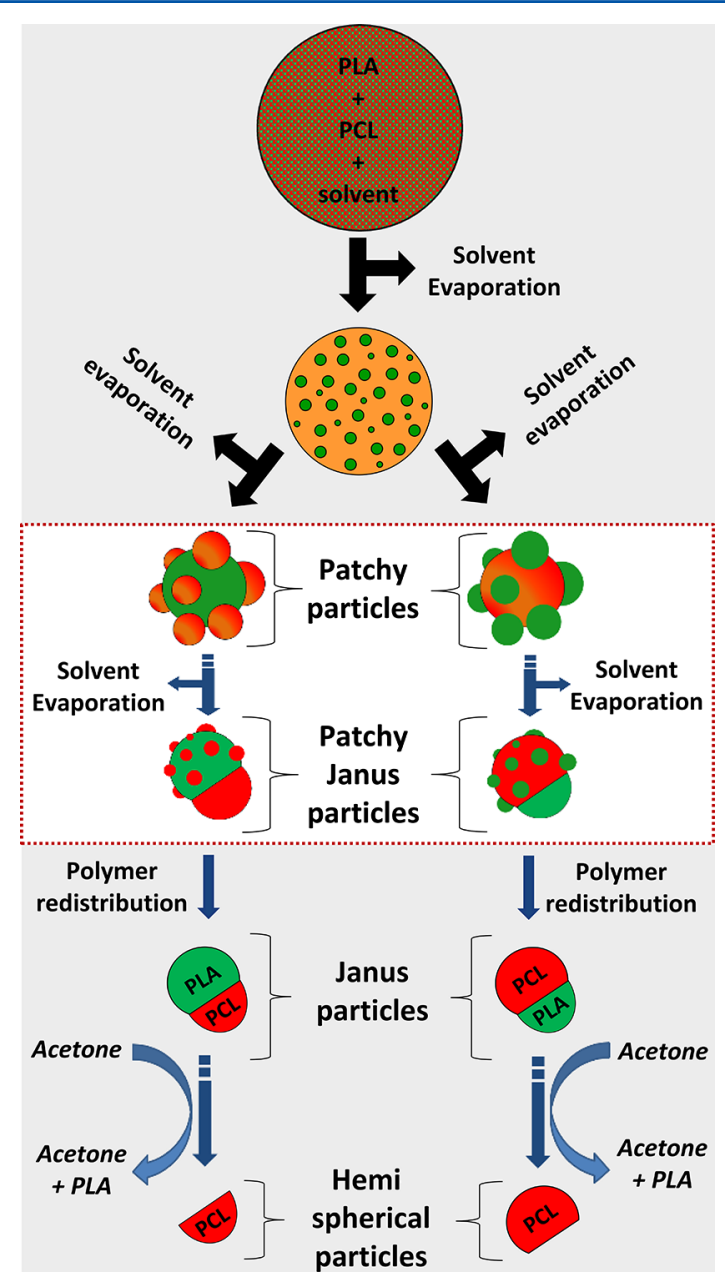

Figure 1. Morphological changes during the evaporation of organic solvent from a droplet composed of a mixture of two polymers and two fluorescent dyes dissolved in a mutually good solvent. The highlighted region is targeted in this study.

mixture of PLA and PCL dissolved in a mutually good solvent. Because of the solvent removal, the concentration of both polymers increases, which eventually leads to polymer-solvent phase separation and the formation of patchy particles, composed of PLA matrix with isolated PCL patches or PCL matrix with PLA patches. The particle morphology depends on the composition of the dispersed phase, $\mathrm{pH}$ of the aqueous phase, ${ }^{15}$ interfacial tension at the contact line, ${ }^{16}$ and spreading coefficients. ${ }^{17}$ Further phase separation leads to the formation of patchy Janus particles composed of two separate polymer domains, with isolated patches of other polymer spread across any of the two regions. Janus particles are formed as a result of full polymer separation, and hemispherical PCL particles can be generated by dissolving PLA domains with acetone. ${ }^{18}$

\section{MATERIALS AND METHODS}

Materials. PCL $\left(M_{\mathrm{w}}=14000 \mathrm{~g} \mathrm{~mol}^{-1}\right.$, Sigma-Aldrich, UK) and PLA $\left(M_{\mathrm{w}}=89000 \mathrm{~g} \mathrm{~mol}^{-1}\right.$, Ingeo 2060D, NatureWorks LLC, Minnetonka, MN, USA) were the main constituents of patchy particles. Dichloromethane (DCM, high-performance liquid chroma- tography grade, Fisher Scientific, UK) and ethyl acetate (EA, SigmaAldrich, UK) were the volatile solvents used to dissolve the polymers. Poly(vinyl alcohol) (PVA, $M_{\mathrm{w}}=13000-23000 \mathrm{~g} \mathrm{~mol}^{-1}, 87-89 \%$ hydrolyzed, Sigma-Aldrich) was used as a hydrophilic stabilizer. Nile red (Sigma-Aldrich, UK) and rhodamine 6G (Rh6G, Sigma-Aldrich, UK) were added to the dispersed phase as fluorescent dyes to visualize the particle structures. 2-[Methoxy(polyethyleneoxy)propyl]-trimethoxysilane (MPEOPS) was used as a wetting agent for the hydrophilic treatment of glass capillaries.

Microfluidic Devices. Single-crystal silicon microchannel (MC) chips consisting of 10 parallel MC arrays (Figure S1a and Table S1) were used to generate relatively small drops by MC (step) emulsification, based on Laplace pressure-driven snap-off. ${ }^{19}$ At the exit of the channels, the dispersed phase expands on the terrace into a flat disk shape (Figure 2a[1],[2]). When the dispersed-phase disk

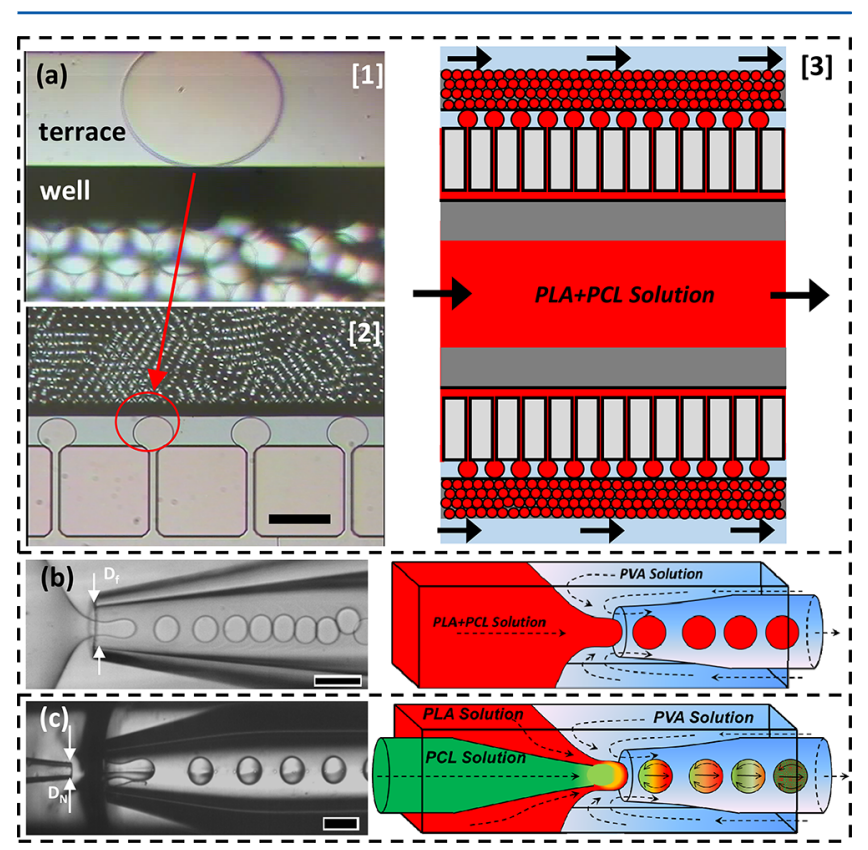

Figure 2. Droplet generation in (a) MC array device; (b) flowfocusing glass capillary device; and (c) coflow-/flow-focusing glass capillary device. Scale bars: (a) 150; (b) 300; and (c) $100 \mu \mathrm{m}$. Experimental conditions: Tables S1 and S2.

reaches the edge of the terrace and enters a deep well, it is no longer confined and expands in three dimensions, assuming a bulbous shape. A higher Laplace pressure of the dispersed phase on the terrace compared to that in the well induces a sudden flow into the well, which rapidly depletes the dispersed phase from the terrace and forms a neck that breaks up via a Rayleigh-Plateau instability. ${ }^{20}$ The drops were removed from the wells by the cross-flowing aqueous phase (Figure 2a[3]).

Glass capillary devices were used to generate large droplets through countercurrent flow focusing (Figure $2 \mathrm{~b}$ and Video S1) or coflow combined with countercurrent flow focusing (Figure $2 \mathrm{c}$ and Video S2). Depending on the device, the dispersed phase was formed by mixing two polymer solutions together before the organic phase was introduced into the device (Figure $2 \mathrm{~b}$ ) or by introducing individual polymer solutions into the device and mixing two separate organic streams in situ, within a formed droplet (Figure 2c). The compositions of the dispersed phase in different devices are shown in Table S2. In all devices, the continuous phase was 5 wt \% aqueous solution of PVA.

Drop Generation in Silicon Chips. A MC chip $(25 \times 28 \times 0.5$ $\mathrm{mm}$, EP Tech, Hitachi, Japan) was assembled in a stainless steel module such that the chip surface was firmly attached to a cover glass to seal the MCs. The drops with a diameter of $25 \mu \mathrm{m}$ were formed using 540 MCs with a width of $18 \mathrm{~m} \mu$ and a height of $5 \mu \mathrm{m}$ (Figure S1b and Videos S3-S5). The drops with a size of $14 \mu \mathrm{m}$ were 
generated using $1840 \mathrm{MCs}$ with a width of $8 \mu \mathrm{m}$ and a height of $4 \mu \mathrm{m}$ (Figure S1c).

Drop Generation in Glass Capillary Devices. Glass capillary devices are coaxial assemblies of borosilicate glass capillaries, fabricated by aligning one or two tapered round capillaries inside a square capillary. ${ }^{21}$ In a two-phase device (Figure 2b), a mixed solution of PLA and PCL was introduced through the outer capillary. In a three-phase device (Figure 2c), a PCL solution was introduced through the injection capillary and a PLA solution was supplied cocurrently through the space between the injection and the outer capillaries. In both cases, the aqueous phase was supplied countercurrently through the space between the collection and outer capillaries. The device was placed on the stage of a GXM XD63 inverted microscope. Live images of droplet generation were recorded at $2000 \mathrm{fps}$ using a Phantom version 9.0 high-speed camera.

Image Analysis and Particle Production. All video recordings were analyzed using ImageJ software to determine the size of the drops (Table S3). The solvent blend was removed from the drops by extraction/evaporation at room temperature.

Confocal Laser Scanning Microscopy Imaging. Particles were stained with Nile red and Rh6G and visualized using a Nikon Eclipse TE300 confocal inverted microscope connected to a computerrunning Zeiss LaserSharp 2000 software. In the flow-focusing device (Figure $2 \mathrm{~b}$ ), both dyes were added to the dispersed phase, which comprised a mixture of PLA and PCL in a DCM/EA blend. In the coflow-/flow-focusing device (Figure 2c), Nile red was added to the middle phase containing EA and PLA while Rh6G, soluble only in DCM, was added to the inner phase containing DCM and PCL. A drop of the sample was placed on a microscope slide and allowed to dry. An argon laser with a wavelength of $488 \mathrm{~nm}$ and a helium-neon laser with a wavelength of $543 \mathrm{~nm}$ were used to excite the samples. For fluorescence detection, two photomultiplier tubes (PMTs) were used: PMT1 captured fluorescence at the $515 \pm 30 \mathrm{~nm}$ range (green region) and PMT2 captured fluorescence above $570 \mathrm{~nm}$ (yellow-red region).

Dye Affinity. The total (Hildebrand) solubility parameter $\left(\delta_{\mathrm{t}}\right)$ for $\mathrm{Rh} 6 \mathrm{G}$ was calculated using the group contribution method according to the following equation 22

$$
\delta_{\mathrm{t}}=\left(\frac{\sum n_{i} E_{\mathrm{coh}_{i}}}{\sum n_{i} V_{\mathrm{m}_{i}}}\right)^{1 / 2}
$$

where $E_{\text {coh }_{i}}$ is the molar cohesive energy, $V_{\mathrm{m}_{i}}$ is the molar volume of $i$ th functional group in Rh6G molecules (Figure S2), and $n_{i}$ is the number of $i$ th functional groups per molecule. The $\delta_{\mathrm{t}}$ values calculated from eq 1 were validated with the empirical $\delta_{\mathrm{t}}$ values from Table 1 .

Table 1. Empirical Values of the Partial Solubility Parameters $\left(\delta_{\mathrm{d}}, \delta_{\mathrm{p}}, \delta_{\mathrm{h}}\right.$ and $\left.\delta_{\mathrm{v}}\right)$ and the Total Solubility Parameters $\left(\delta_{\mathrm{t}}\right)$ for PLA, PCL, and Rh6G

$\begin{array}{crrr}a_{\text {solubility parameters }}\left(\mathrm{J} \mathrm{cm}^{-3}\right)^{1 / 2} & \mathrm{PLA}^{b} & \mathrm{PCL}^{b} & \mathrm{Rh}^{b} \mathrm{G}^{c} \\ \delta_{\mathrm{d}} & 17.6 & 17.0 & 20.0 \\ \delta_{\mathrm{p}} & 9.7 & 4.8 & 14.3 \\ \delta_{\mathrm{h}} & 11.8 & 8.3 & 10.2 \\ \delta_{\mathrm{v}}=\sqrt{\delta_{\mathrm{d}}{ }^{2}+\delta_{\mathrm{p}}{ }^{2}} & 20.1 & 17.7 & 24.6 \\ \delta_{\mathrm{t}}=\sqrt{\delta_{\mathrm{d}}{ }^{2}+\delta_{\mathrm{p}}{ }^{2}+\delta_{\mathrm{h}}{ }^{2}} & 23.3 & 19.5 & 26.7\end{array}$

${ }^{a} \delta$ = partial solubility parameters; the subscripts $\mathrm{d}$, $\mathrm{p}$, and $\mathrm{h}$ denote the contribution of dispersion forces, polar forces, and hydrogen bonding; and $\mathrm{v}$ combines volume-dependent dispersion and polar forces and $\mathrm{t}$ is the total contribution. ${ }^{b}$ The partial solubility parameters of the polymers taken from Othman et al. ${ }^{23}{ }^{c}$ The partial solubility parameters of the dye taken from Hansen. ${ }^{24}$

\section{RESULTS AND DISCUSSION}

Dye Affinity. Table 1 gives the empirical values of the total solubility parameters for PLA, PCL, and Rh6G from the literature.

As shown in Supporting Information S4, the calculated $\delta_{\mathrm{t}}$ value for Rh6G was $25.5\left(\mathrm{~J} / \mathrm{cm}^{3}\right)^{0.5}$, which was very close to the empirical value of $26.7\left(\mathrm{~J} / \mathrm{cm}^{3}\right)^{0.5}$ from Table 1. The closer proximity of both $\delta_{\mathrm{t}}$ values of Rh6G to PLA (23.3) than PCL (19.5) indicates the preferential sorption of Rh6G on PLA over PCL. This conclusion was confirmed using a Bagley's graph shown in Figure S3. Rh6G shows a good solubility in the polymer if the following condition is satisfied as in Figure $\mathrm{S}^{21}$

$$
5>\sqrt{\left(\delta_{\mathrm{v}, \text { RhGG }}-\delta_{\mathrm{v}, \mathrm{P}}\right)+\left(\delta_{\mathrm{h}, \text { RhGG }}-\delta_{\mathrm{h}, \mathrm{P}}\right)^{2}}
$$

This condition was satisfied for PLA and not satisfied for PCL, indicating that only PLA is a good solvent for Rh6G. The preferential affinity of Rh6G for PLA was confirmed by confocal laser scanning microscopy (CLSM) images of polymer films stained with the dyes, shown in Supporting Information S4. Rh6G-stained PCL films do not show green fluorescence (insets (b.2) and (d.2) in Figure S4), indicating that Rh6G (alone or in combination with Nile red) is not soluble in PCL. However, Rh6G-containing PLA films show green fluorescence on PMT1 images (insets (a.2) and (c.2)), clearly indicating that Rh6G has a high affinity to PLA.

Internal Phase Separation. When two immiscible polymers, PLA and PCL, are dissolved in a mutually good organic solvent or solvent blend and dispersed in an aqueous phase, they will be fully mixed within the formed droplets because of the entropic gain $\left(\Delta S_{\mathrm{M}}>0\right)$ due to the random distribution of the polymer chains and favorable solventpolymer interactions $\left(\Delta H_{\mathrm{M}}<0\right)$, which results in a negative Gibbs free energy of mixing, $\Delta G_{\mathrm{M}}$, where $\Delta G_{\mathrm{M}}=\Delta H_{\mathrm{M}}-$ $T \Delta S_{\mathrm{M}}$. As the solvent is removed, the concentration of both polymers increases and the entropic gain becomes insufficient to overcome the enthalpic penalty $\left(\Delta H_{\mathrm{M}}>0\right)$ due to the disfavored PCL-PLA interactions, which results in a spontaneous phase separation via spinodal decomposition. As a result, small domains of PCL-rich phases surrounded by the domains of PLA-rich phases are formed. ${ }^{15}$ Further solvent removal causes phase inversion and the formation of discrete spherical particles (patches) of one polymer-rich phase embedded in a continuous matrix of the other.

Ostwald Ripening. In the last stage, known as Ostwald ripening or coarsening, the polymer diffuses away from smaller patches and the dissolved polymer deposits onto larger patches to reduce the overall interfacial free energy of the system. As a result, larger patches grow at the expense of smaller ones, which eventually disappear, and the total number of patches in the continuous polymer phase is reduced with time. The process ends with full polymer separation and the formation of Janus (bifacial) particles with two separate polymer domains.

If the polymer bulk diffusion through the matrix is slower than the surface diffusion, the average radius of patches, $\langle R\rangle$, increases with time, $t$, according to the Lifshitz-Slyozov theory $^{25}$

$$
\langle R\rangle-\langle R\rangle_{0}^{3}=\frac{8 \gamma c_{\infty} v^{2} D}{9 R_{\mathrm{g}} T} t
$$

where $\langle R\rangle_{0}$ is the average radius of patches at zero time, $\gamma$ is the interfacial tension at the patch/matrix boundary, $c_{\infty}$ is the 
solubility of the polymer at a flat interface, $v$ is the molar volume of the polymer, $D$ is the diffusion coefficient of the polymer, $R_{\mathrm{g}}$ is the ideal gas constant, and $T$ is the absolute temperature. Therefore, the coarsening rate critically depends on the diffusivity of the dissolved polymer through the particle matrix, which is determined by the diffusion coefficient, $D$.

Generation of Patchy Particles. To produce patchy particles, highlighted in Figure 1, Ostwald ripening must be suppressed by minimizing $D$, which can be achieved by the fast removal of the solvent(s) from the droplets, which will increase the viscosity within the phase-separated drops. Alternatively, the solubility $c_{\infty}$ of the diffusing polymer can be reduced by using another solvent in which the polymer is less soluble.

One approach to increase the rate of solvent removal from the drops was to dissolve the polymers in DCM/EA blend rather than in pure DCM. EA is almost 5 times more soluble in water than DCM $\left(83 \mathrm{~g} / \mathrm{L}\right.$ vs $17.5 \mathrm{~g} / \mathrm{L}$ at $\left.20^{\circ} \mathrm{C}\right)$, and thus, EA was removed faster from the drops by dissolution into the aqueous phase. Indeed, the degree of polymer separation was reduced by increasing the EA/DCM ratio in the dispersed phase from 0:100 to 25:75 to 50:50 to 75:25 (Figure 3i-iv).

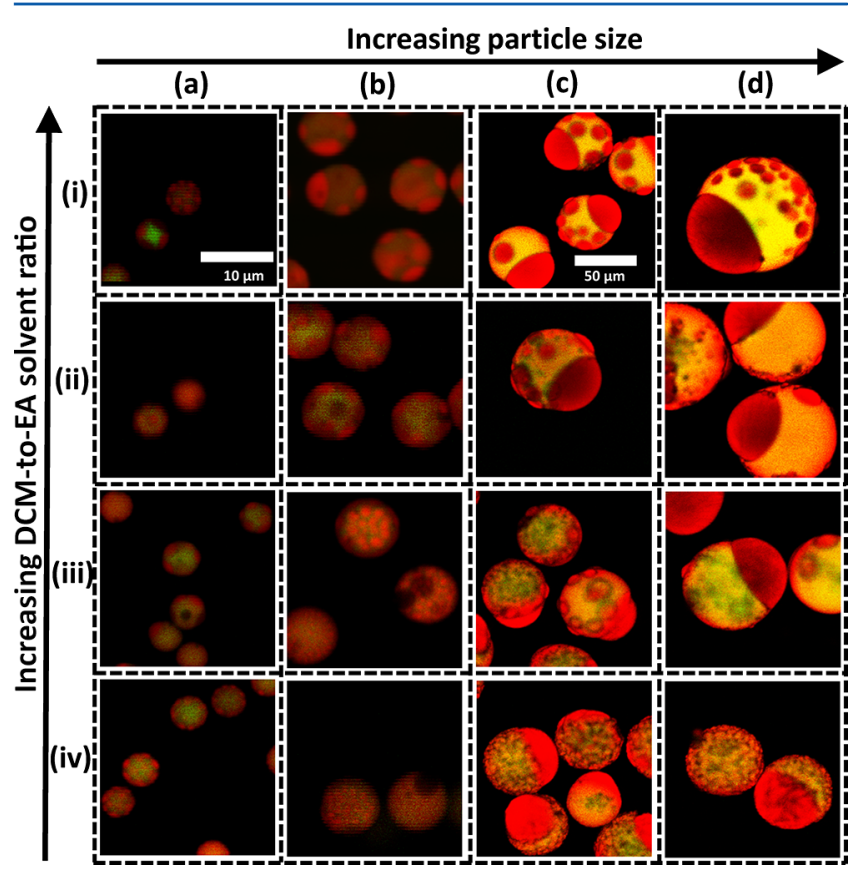

Figure 3. CLSM images of particles formed using (a) step emulsification with $4 \mu \mathrm{m}$ depth MCs; (b) step emulsification with 5 $\mu \mathrm{m}$ depth MCs; and (c,d) flow-focusing glass capillary devices. DCM/ EA ratios in the organic phase: (i) 100:0; (ii) 75:25; (iii) 50:50; and (iv) 25:75. The PLA/PCL ratio was 2:1 (v/v) in all cases. Particle diameters: (a) 4.5; (b) 7.4; (c) 50-70; and (d) 74-105 $\mu \mathrm{m}$. Scale bars: $(\mathrm{a}, \mathrm{b}) 5$ and $(\mathrm{c}, \mathrm{d}) 50 \mu \mathrm{m}$. Yellow or green patches = PLA and red patches $=$ PCL

The second approach to increase the rate of the solvent removal and prevent full polymer separation was to reduce the droplet size using step emulsification devices (Figure 3a,b), instead of glass capillary devices used in Figure 3c,d. For all solvent blends, the degree of polymer separation was reduced by decreasing the droplet size from 74-105 $\mu \mathrm{m}$ (Figure 3d) to $4.5 \mu \mathrm{m}$ (Figure 3a). This occurred due to the increased surface area-to-volume ratio of small droplets. The size of the drops produced by step emulsification barely depends on the emulsion formulation and the flow rates of the dispersed and continuous phases below a critical capillary number and is mainly determined by the geometry of the device, such as the width and height of the MCs and the length of the terrace. ${ }^{20}$ Therefore, all particles produced by step emulsification using the same geometry of the MCs had the same size, irrespective of the solvent ratio, which was $4.5 \mu \mathrm{m}$ (Figure $3 \mathrm{a}$ ) or $7.4 \mu \mathrm{m}$ (Figure $3 \mathrm{~b}$ ) for the height of the MCs of 4 and $5 \mu \mathrm{m}$, respectively.

Small particles made using step emulsification were obtained with a symmetric shape and numerous PCL patches embedded in a PLA matrix. The minor polymer constituent (PCL) forms a discontinuous phase, and the major constituent (PLA) forms a continuous matrix. Because of the small droplet size and high aqueous-to-organic flow rate ratio of 100 , the solvent was rapidly removed from the drops, which brought the polymer migration to a standstill and prevented the formation of Janus structure. The size of PCL patches increased from Figure $3 b$ (iv) to $3 b(i)$ because of the lower removal rate of DCM as compared to EA. It should be noted that Ostwald ripening was more suppressed in the presence of EA because it is the poorer solvent for PCL than DCM. In Figure 3, the polymer ratio was constant, and thus, the number of patches per particle was proportional to $\left\langle R_{\mathrm{p}}\right\rangle^{-3}$, where $\left\langle R_{\mathrm{p}}\right\rangle$ is the average diameter of the patches.

Patchy particles can be used for programmable drug delivery $^{26}$ because the size and number of patches can be precisely tuned and PCL and PLA domains exhibit distinct binding affinities to various drugs and different biodegradation rates. In addition, the patchy particles shown in Figure $3 \mathrm{a}$ are smaller than the size of red blood cells $(6-8 \mu \mathrm{m})$ and can be injected directly into the bloodstream by intravenous administration. Patchy particles with precise arrangement of patches and patch "recognition" ability can also be used as building blocks for the fabrication of self-assembled 3-D structures. $^{27}$

Generation of Patchy Janus Particles. The particles produced in glass capillary devices had an asymmetric structure with a patchy Janus morphology (Figure 3c,d) because of the longer droplet consolidation times associated with their much larger size. The size of the drops produced by flow focusing was varied widely from 50 to $105 \mu \mathrm{m}$ in accordance with the scaling law: ${ }^{28} D_{\mathrm{d}} / D_{\mathrm{f}}=0.23\left(Q_{\mathrm{c}} / Q_{\mathrm{d}}\right)^{-0.39}$, where $Q_{\mathrm{d}}$ is the dispersed phase flow rate, $Q_{c}$ is the continuous phase flow rate, $D_{\mathrm{d}}$ is the droplet diameter, and $D_{\mathrm{f}}$ is the orifice diameter shown in Figure $2 b$. The most complete polymer separation was observed in Figure $3 \mathrm{~d}(\mathrm{i}, \mathrm{ii})$, for particles with a diameter above $100 \mu \mathrm{m}$ prepared using high DCM/EA ratios.

The effect of solvent ratio on the particle morphology for a large population of patchy particles is shown in Figure 4. The CLSM images of the particles are shown in Figure 3c(ii,iv). The most opaque particles were obtained using the lowest DCM-toEA ratio (Figure 4c) because of the minimum degree of polymer separation achieved and the presence of numerous tiny patches in the particle matrix that caused intense light scattering. On the other hand, the most transparent particles were obtained using the highest amount of DCM in the blend (Figure $4 \mathrm{a}$ ) because of the maximum degree of polymer separation. In addition, the particles produced from the 50:50 solvent blend (Figure 4b) had much larger patches than those produced from the blend containing 25\% DCM and 75\% EA (Figure 4c). 

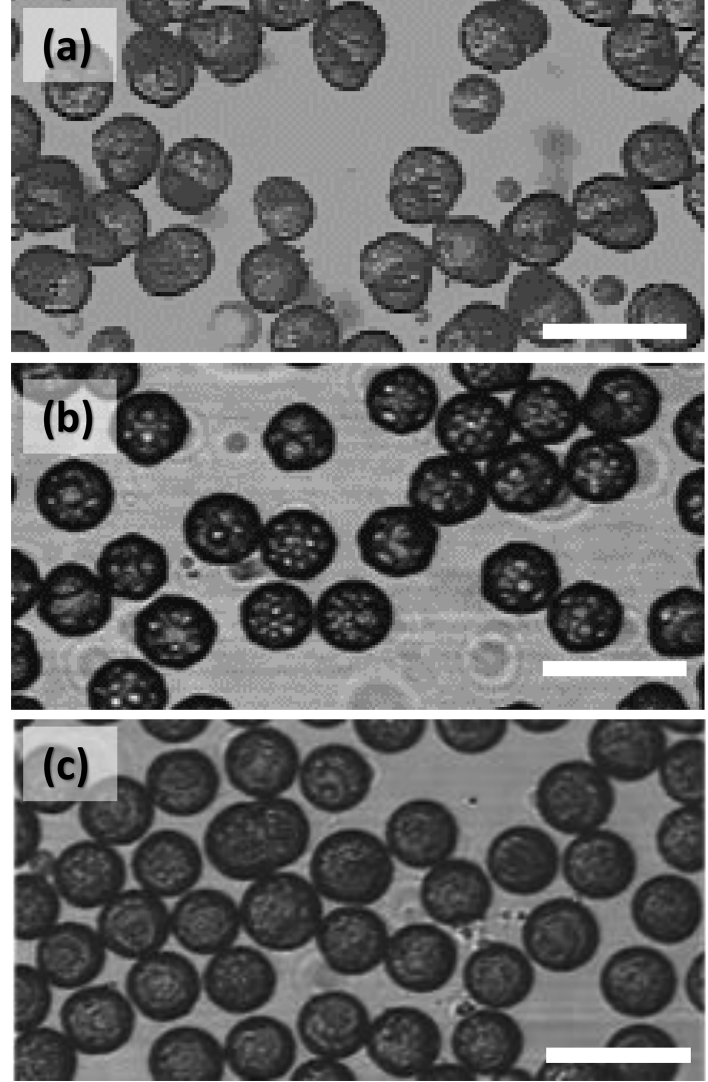

Figure 4. Optical micrographs of patchy particles formed using flowfocusing glass capillary device. DCM/EA ratio in the organic phase: (a) 75:25; (b) 50:50; and (c) 25:75. Scale bar: $100 \mu \mathrm{m}$ for all images.

Generation of Janus Particles. The particles shown in Figure $3 c$,d have imperfect Janus morphologies, with the PCL patches distributed across the PLA compartments and vice versa. To improve polymer separation during the particle consolidation, the two polymers were dissolved in different organic solvents and delivered as separate streams (Figure 2c). These two incoming polymer streams were merged together downstream of the injection capillary and formed drops with combined contents of both streams. If both polymers have the same affinity to both solvents, the contents of the two streams will be efficiently mixed within a newly formed drop because of the internal turbulent eddies as a result of vortex flow during drop pinch-off. ${ }^{18,29}$ However, if one of the two polymers is miscible in the carrier solvent and only partially miscible in the other, the two streams would tend to stay separated.

To demonstrate the applicability of this approach, a solution composed of 3 wt \% PCL in DCM was delivered through the injection capillary, while a solution composed of $3 \mathrm{wt} \%$ PLA in EA was supplied cocurrently through the pockets between the injection and outer capillaries. Because PCL is poorly soluble in $\mathrm{EA}$, the two streams tend to stay separated within the formed drop, with the DCM stream occupying the lower hemisphere of the Janus drops due to the higher density of DCM compared to EA. As shown in Figure 2c, the two streams were not fully mixed within the droplets in the collection capillary.

Figure 5 shows a comparison of Janus particles formed by premixing and in situ mixing of $3 \mathrm{wt} \%$ PLA in EA solution and 3 wt \% PCL in DCM solution. The Janus particles formed via the premixing route were patchier (Figure $5 \mathrm{a}-\mathrm{c}$ ) as compared to those produced via in situ mixing, which had almost perfect Janus morphologies (Figure $5 \mathrm{~d}-\mathrm{f}$ ). It reflects the fact that in the case of in situ mixing, PLA and PCL were not homogeneously dispersed in the drops after their generation, whereas in the case of premixing, the polymers have been uniformly distributed in the droplets. The volume fractions of the two polymer compartments were varied continuously in a facile manner during the drop generation, by simply readjusting the flow rates of the two polymer solutions, which could otherwise have been achieved via batch-wise premixing of the solutions but separately for each polymer ratio. As shown in Figure 5, the variation in PLA-to-PCL flow rate ratio from 2:1 to $1: 1$ to $1: 2$ led to corresponding changes in the size of PCL and PLA compartments in the resultant Janus particles. For example, the smallest size of PLA compartments was achieved using the minimum PLA-to-PCL ratio of 1:2 (Figure $5 \mathrm{f}(\mathrm{iii})$ ). It should be also noted that the variations of fluid flow rates which led to the reduced PLA content in the blend also led to the

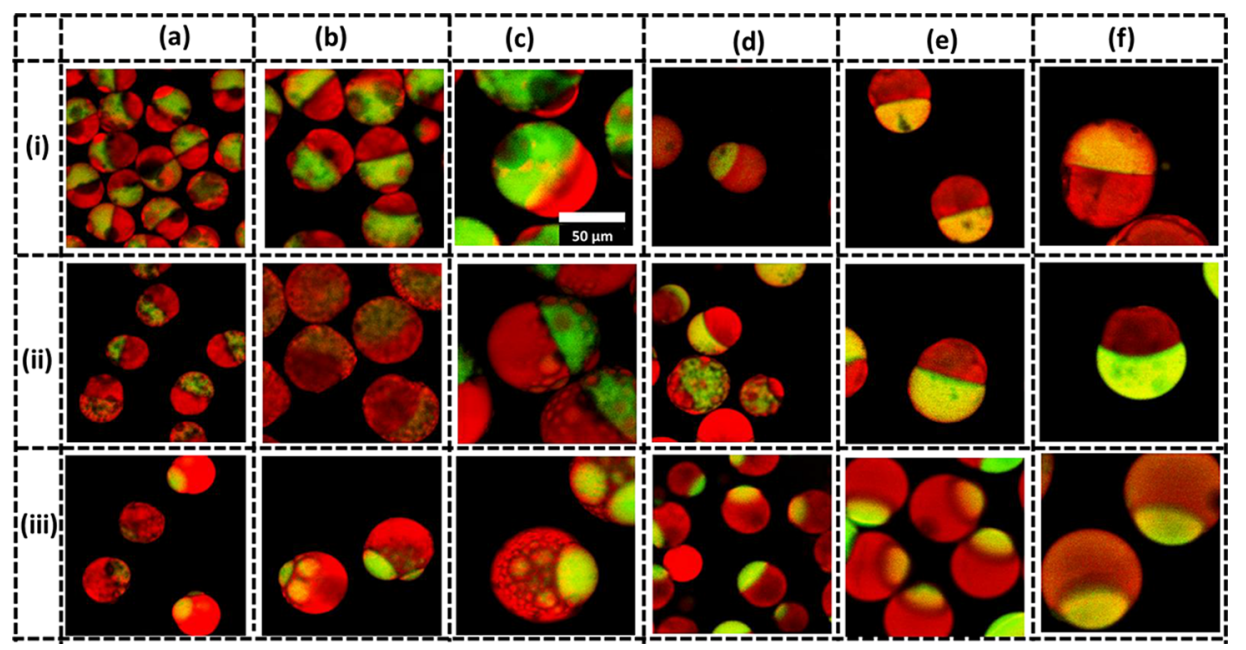

Figure 5. CLSM images of particles formed using $(a-c)$ premixed polymer solutions and $(d-f)$ in situ mixed polymer solutions. PLA/PCL ratio (v/ v): (i) 2:1, (ii) 1:1, and (iii) 1:2. PLA/PCL ratios for (d-f) were achieved by flow rate ratio adjustments (Table S1). Particle diameters: (a) 32-33; (b) 48-54 ; (c) 79-81; (d) 40-41; (e) 51-63; and (f) 75-87 $\mu \mathrm{m}$. Scale bar: $50 \mu \mathrm{m}$ on c(i) represents all images. Yellow-green patches $=$ PLA and red-brown patches $=$ PCL. 
reduced EA content, which caused more complete polymer separation at the lower PLA/PCL ratios. In addition, the particles with the fixed polymer ratio produced in the same device showed different degrees of polymer separation because of their different sizes. For example, the particles with a polymer ratio of 1:1 showed an increasing level of polymer separation from Figure $5 \mathrm{~d}$ to $\mathrm{f}$ as their sizes increased from Figure $5 \mathrm{~d}$ to $\mathrm{f}$.

\section{CONCLUSIONS}

Bipolymer particles composed of PCL and PLA domains have been produced with tuneable size, composition, morphology, and shape anisotropy using microfluidic emulsification and solvent evaporation. The particle morphology was observed by CLSM using two fluorescent dyes with different binding affinities to the constituent polymers. The particle morphology was controlled by changing the organic phase composition, the size of the droplets, and the fabrication route.

Isotropic patchy particles were formed from relatively small drops, whereas anisotropic patchy Janus particles were obtained from larger drops due to longer particle solidification times. For solvents used in this study, all particles smaller than $10 \mu \mathrm{m}$ showed a patchy morphology, whereas the particles larger than $30 \mu \mathrm{m}$ had a bifacial (Janus) morphology. The size and number of patches in the particle matrix can be tuned via the manipulation of the solvent ratio and droplet size, which had a profound effect on the rate of Ostwald ripening in the late stages of polymer separation. More complete polymer separation resulted in smaller number of larger patches, which was achieved at the higher DCM-to-EA ratio in the organic phase. Janus particles with two almost fully separated polymer compartments were produced by mixing two separate polymer streams in situ within the formed drops. An added benefit of this approach is that the ratio of the two polymers and solvents can be continuously changed during the drop generation, simply by manipulating the flow rates of the two organic streams in the microfluidic device.

Bipolymer particles with controlled patchiness and shape anisotropy can be exploited for dual-site drug delivery and a simultaneous release of two incompatible drugs showing markedly different solubilities in the two polymers. The loading capacity and release rate of the drugs can be adjusted through controlling the size of different polymer domains in the particle and the size of the whole particle.

\section{ASSOCIATED CONTENT}

\section{S Supporting Information}

The Supporting Information is available free of charge on the ACS Publications website at DOI: 10.1021/acs.langmuir.7b02506.

Geometry of the MC chips, compositions of the fluid streams used for the production of particles, information about the supporting videos, and calculation of the total solubility parameter for Rh6G and confocal microscopic images of polymer films stained with the dyes (PDF)

Large droplets generated through countercurrent flow focusing (AVI)

Large droplets generated through coflow combined with countercurrent flow focusing (AVI)

Droplet pinch-off from terrace for droplet generation in $\mathrm{MC}$ array device (AVI)
Monodisperse droplet generation across terraces in $\mathrm{MC}$ array device (AVI)

Generated droplet flow in cross flowing aqueous phase of MC array device (AVI)

\section{AUTHOR INFORMATION}

\section{Corresponding Author}

*E-mail: G.Vladisavljevic@lboro.ac.uk.

ORCID $\odot$

Goran T. Vladisavljević: 0000-0002-8894-975X

\section{Author Contributions}

The manuscript was written through the contributions of all authors. All authors have given approval to the final version of the manuscript.

\section{Notes}

The authors declare no competing financial interest.

\section{ACKNOWLEDGMENTS}

E.E.E. holds a scholarship from Niger Delta Development Commission (NDDC), Nigeria. The authors gratefully acknowledge the financial support from the EPSRC grant $\mathrm{EP} / \mathrm{HO} 29923 / 1$.

\section{ABBREVIATIONS}

CLSM,confocal laser scanning microscopy; DCM,dichloromethane; EA,ethyl acetate; MPEOPS,2-[methoxy(polyethyleneoxy)propyl]-trimethoxysilane; PCL,polycaprolactone; PLA,poly(DL-lactic acid); PVA,poly(vinyl alcohol); Rh6G,rhodamine 6G

\section{REFERENCES}

(1) Knight, J. B.; Vishwanath, A.; Brody, J. P.; Austin, R. H. Hydrodynamic Focusing on a Silicon Chip: Mixing Nanoliters in Microseconds. Phys. Rev. Lett. 1998, 80, 3863-3866.

(2) Jen, C.-P.; Wu, C.-Y.; Lin, Y.-C.; Wu, C.-Y. Design and Simulation of the Micromixer with Chaotic Advection in Twisted Microchannels. Lab Chip 2003, 3, 77.

(3) Khan, S. A.; Jensen, K. F. Microfluidic Synthesis of Titania Shells on Colloidal Silica. Adv. Mater. 2007, 19, 2556-2560.

(4) Seemann, R.; Brinkmann, M.; Pfohl, T.; Herminghaus, S. Droplet Based Microfluidics. Rep. Prog. Phys. 2012, 75, 16601.

(5) Kim, J. H.; Jeon, T. Y.; Choi, T. M.; Shim, T. S.; Kim, S.-H.; Yang, S.-M. Droplet Microfluidics for Producing Functional Microparticles. Langmuir 2014, 30, 1473-1488.

(6) Watanabe, T.; Lopez, C. G.; Douglas, J. F.; Ono, T.; Cabral, J. T. Microfluidic Approach to the Formation of Internally Porous Polymer Particles by Solvent Extraction. Langmuir 2014, 30, 2470-2479.

(7) Baldelli, A.; Boraey, M. A.; Nobes, D. S.; Vehring, R. Analysis of the Particle Formation Process of Structured Microparticles. Mol. Pharm. 2015, 12, 2562-2573.

(8) Zhu, J.; Hayward, R. C. Hierarchically Structured Microparticles Formed by Interfacial Instabilities of Emulsion Droplets Containing Amphiphilic Block Copolymers. Angew. Chem., Int. Ed. 2008, 47, 2113-2116.

(9) McConnell, M. D.; Kraeutler, M. J.; Yang, S.; Composto, R. J. Patchy and Multiregion Janus Particles with Tunable Optical Properties. Nano Lett. 2010, 10, 603-609.

(10) Ling, X. Y.; Phang, I. Y.; Acikgoz, C.; Yilmaz, M. D.; Hempenius, M. A.; Vancso, G. J.; Huskens, J. Janus Particles with Controllable Patchiness and Their Chemical Functionalization and Supramolecular Assembly. Angew. Chem., Int. Ed. 2009, 48, 7677-7682.

(11) Kim, J. W.; Cho, J.; Cho, J.; Park, B. J.; Kim, Y.-J.; Choi, K.-H.; Kim, J. W. Synthesis of Monodisperse Bi-Compartmentalized 
Amphiphilic Janus Microparticles for Tailored Assembly at the OilWater Interface. Angew. Chem., Int. Ed. 2016, 55, 4509.

(12) Wang, X.; Miller, D. S.; de Pablo, J. J.; Abbott, N. L. Reversible Switching of Liquid Crystalline Order Permits Synthesis of Homogeneous Populations of Dipolar Patchy Microparticles. Adv. Funct. Mater. 2014, 24, 6219-6226.

(13) Hirai, Y.; Wakiya, T.; Yabu, H. Virus-Like Particles Composed of Sphere-Forming Polystyrene-Block-Poly(t-Butyl Acrylate) (PS-bPtBA) and Control of Surface Morphology by Homopolymer Blending. Polym. Chem. 2017, 8, 1754-1759.

(14) Anderson, J. M.; Shive, M. S. Biodegradation and Biocompatibility of PLA and PLGA Microspheres. Adv. Drug Delivery Rev. 1997, $28,5-24$.

(15) Min, N. G.; Ku, M.; Yang, J.; Kim, S.-H. Microfluidic Production of Uniform Microcarriers with Multicompartments through Phase Separation in Emulsion Drops. Chem. Mater. 2016, 28, 1430-1438.

(16) Wei, D.; Ge, L.; Lu, S.; Li, J.; Guo, R. Janus Particles Templated by Janus Emulsions and Application as a Pickering Emulsifier. Langmuir 2017, 33, 5819-5828.

(17) Cao, X.; Li, W.; Ma, T.; Dong, H. One-Step Fabrication of Polymeric Hybrid Particles with Core-shell, Patchy, Patchy Janus and Janus Architectures via a Microfluidic-Assisted Phase Separation Process. RSC Adv. 2015, 5, 79969-79975.

(18) Ekanem, E. E.; Nabavi, S. A.; Vladisavljević, G. T.; Gu, S. Structured Biodegradable Polymeric Microparticles for Drug Delivery Produced Using Flow Focusing Glass Microfluidic Devices. ACS Appl. Mater. Interfaces 2015, 7, 23132-23143.

(19) Sugiura, S.; Nakajima, M.; Kumazawa, N.; Iwamoto, S.; Seki, M. Characterization of Spontaneous Transformation-Based Droplet Formation During Microchannel Emulsification. J. Phys. Chem. B 2002, 106, 9405-9409.

(20) Huang, X.; Eggersdorfer, M.; Wu, J.; Zhao, C.-X.; Xu, Z.; Chen, D.; Weitz, D. A. Collective Generation of Milliemulsions by StepEmulsification. RSC Adv. 2017, 7, 14932-14938.

(21) Shum, H. C.; Lee, D.; Yoon, I.; Kodger, T.; Weitz, D. A. Double Emulsion Templated Monodisperse Phospholipid Vesicles. Langmuir 2008, 24, 7651-7653.

(22) Karst, D.; Yang, Y. Using the Solubility Parameter to Explain Disperse Dye Sorption on Polylactide. J. Appl. Polym. Sci. 2005, 96, $416-422$.

(23) Othman, R.; Vladisavljević, G. T.; Bandulasena, H. C. H.; Nagy, Z. K. Production of Polymeric Nanoparticles by Micromixing in a CoFlow Microfluidic Glass Capillary Device. Chem. Eng. J. 2015, 280, 316-329.

(24) Hansen, C. M. Hansen Solubility Parameters: A User's Handbook, 2nd ed.; CRC Press: Boca Raton, 2007.

(25) Lifshitz, I. M.; Slyozov, V. V. The Kinetics of Precipitation from Supersaturated Solid Solutions. J. Phys. Chem. Solids 1961, 19, 35-50.

(26) Li, W.; Dong, H.; Tang, G.; Ma, T.; Cao, X. Controllable Microfluidic Fabrication of Janus and Microcapsule Particles for Drug Delivery Applications. RSC Adv. 2015, 5, 23181-23188.

(27) Zhang, Z.; Glotzer, S. C. Self-Assembly of Patchy Particles. Nano Lett. 2004, 4, 1407-1413.

(28) Vladisavljević, G. T.; Duncanson, W. J.; Shum, H. C.; Weitz, D. A. Emulsion Templating of Poly(lactic acid) Particles: Droplet Formation Behavior. Langmuir 2012, 28, 12948-12954.

(29) Song, H.; Bringer, M. R.; Tice, J. D.; Gerdts, C. J.; Ismagilov, R. F. Experimental Test of Scaling of Mixing by Chaotic Advection in Droplets Moving through Microfluidic Channels. Appl. Phys. Lett. 2003, 83, 4664-4666. 